\title{
Very late stent thrombosis in everolimus-eluting stent with predisposing mechanical factors: Differential features
}

\author{
Milosz Jaguszewski ${ }^{1,2,3 *}$, Carlos Cortés ${ }^{1,4 *}$, Halyna Daucher ${ }^{1}$, Michele Schincariol ${ }^{2}$, \\ Michal Halejcio ${ }^{5}$, Petra Besuch ${ }^{5}$, Juan Luis Gutiérrez-Chico ${ }^{1,2}$ \\ ${ }^{1}$ Interventional Cardiology, Klinikum Frankfurt (Oder), Germany \\ ${ }^{2}$ Institute of Cardiovascular Translational Research of Atlantic (ICTRA), Berlin, Germany \\ ${ }^{3}$ First Department of Cardiology, Medical University of Gdansk, Poland \\ ${ }^{4}$ Interventional Cardiology Department, Hospital Clínico Universitario de Valladolid, ICICOR, Valladolid, Spain \\ ${ }^{5}$ Institute of Pathology, Klinikum Frankfurt (Oder), Germany
}

This paper was guest edited by Prof. Nicolas Foin

\section{Introduction}

The clinical meaning of acute malapposition and of the presence of struts detached from the coronary wall is still unclear. It has been demonstrated that the healing process of jailing and malapposed struts is delayed with respect to well-apposed ones [1,2]. Moreover, the risk of malapposed struts to remain uncovered is directly proportional to the magnitude of the detachment: the further the strut from the vessel wall, the more likely to remain uncovered [2], the most severe the fluid disturbances induced [3] and the higher the shear stress rate on the strut surface, that might have a prothrombotic effect [3]. Nonetheless there is no compelling evidence hitherto linking these pathophysiologic and biomechanical phenomena in malapposed and jailing struts with an adverse clinical outcome, namely with a higher incidence of thrombotic events, therefore the question about how far the stent optimisation should be driven remains open. Hereby we are reporting to the best of our knowledge the first case of very late stent thrombosis (VLST) most likely due to suboptimal stent deployment and the subsequent disturbances in coronary flow and biomechanics, exhibiting some differential features as compared with VLST in other anatomic scenarios [4-6], thus strongly suggesting a differentiated pathophysiologic mechanism.

\section{Case report}

A 64-year-old female patient, smoker, was admitted to the emergency room for recurrent chest pain during the last $12 \mathrm{~h}$. Drug-eluting stents (DES) had been implanted in the proximal left anterior descending artery (LAD) (> 100 months ago) and in the ostial left circumflex artery (LCx) (31 months ago) according to an external report, but no more information was available. The electrocardiogram showed negative $\mathrm{T}$ waves in precordial leads and serum cardiac markers were elevated. Notwithstanding optimal medical treatment for non-ST-elevation myocardial infarction, intermittent and recurrent episodes of angina prompted an urgent coronary angiography 90 min after admission, that unravelled a waving intraluminal filling defect in the proximal LAD (Fig. 1; Video 1 - see journal website, supplementary file).

Optical coherence tomography (OCT) showed a stent in the proximal LAD, covered by a homogeneous and regular layer, highly suggestive of neointima (Fig. 2A; Video $2-$ see journal website, supplementary file), whilst the stent in the ostial LCx extended to the left main

Address for correspondence: Prof. Juan Luis Gutiérrez-Chico, MD, PhD, FESC, FACC, Head of Interventional Cardiology, Klinikum Frankfurt (Oder), Müllroser Chaussée 7, 15236 - Frankfurt (Oder), Germany, tel: +49 (0) 17630585019 , +34615 319370, e-mail: juanluis.gutierrezchico@ictra.es

*Equally contributed 


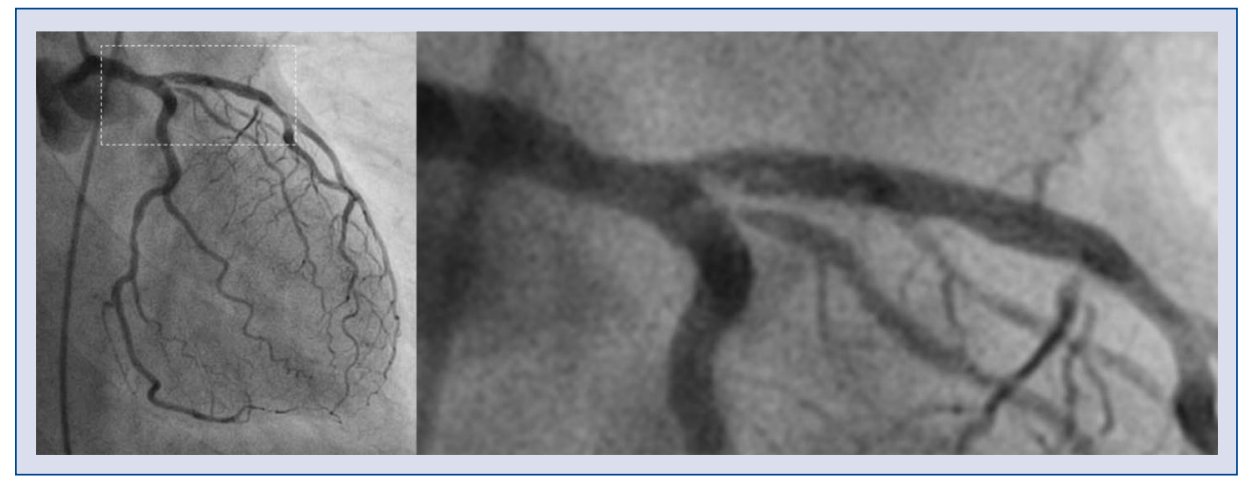

Figure 1. Coronary angiography, right anterior oblique caudal projection, showing a waving intraluminal filling defect in the proximal left anterior descending artery (Video 1 - see journal website, supplementary file).

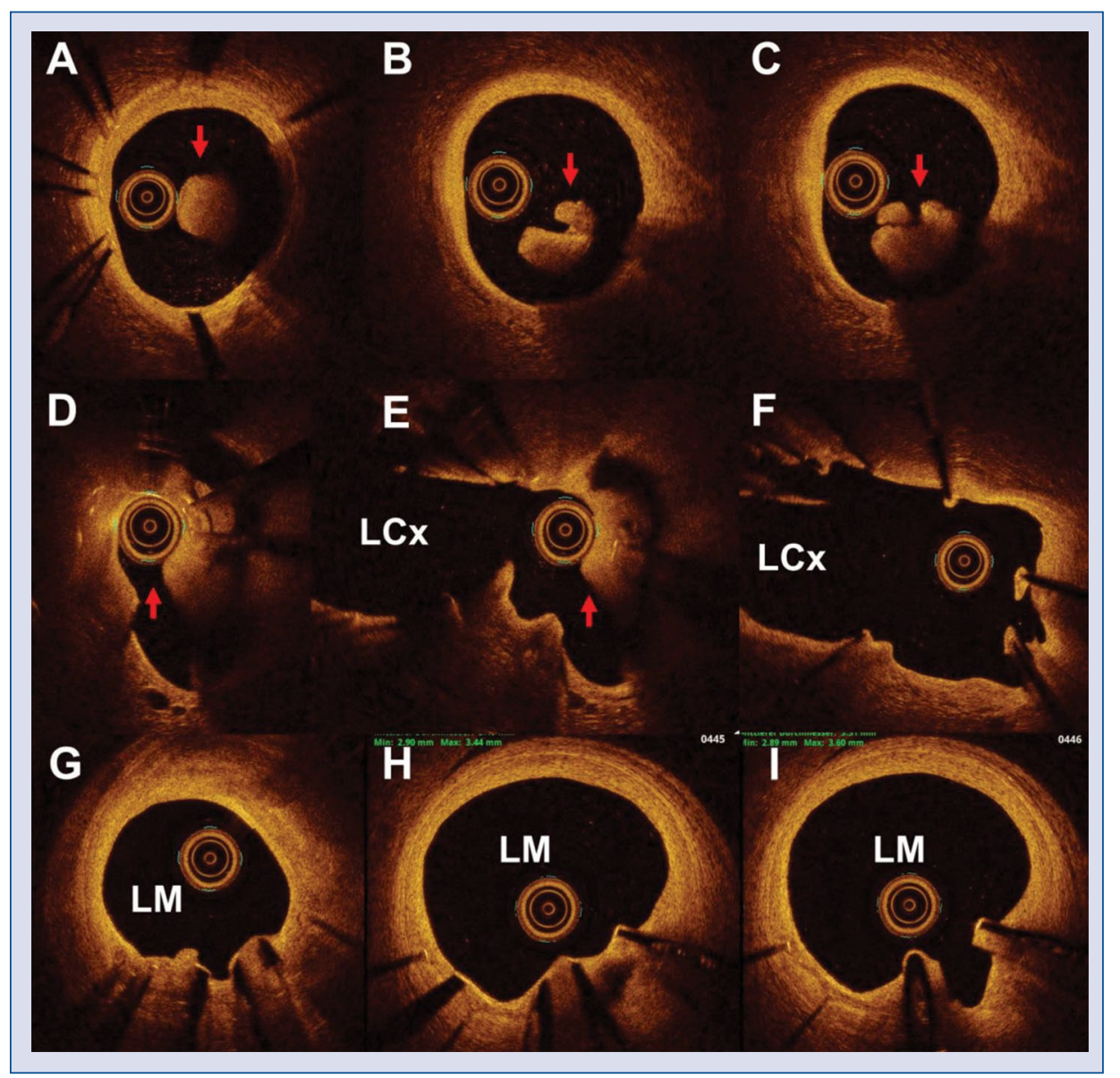

Figure 2. Optical coherence tomography cross-sections, showing a red thrombus (A-E, red arrows), attached to the jailing struts (D, E) and floating in the left anterior descending artery (LAD) (A-C). The stent in the left circumflex artery (LCX) extends to the left main artery (LM), jailing the ostium of the LAD (D-F). Its proximal part is crushed against the LM wall, showing a double metallic layer on one sector, whilst no metallic layer in the opposite sectors (G-I).

artery (LM), thus jailing the ostium of the LAD (Fig. 2; Video 2 - see journal website, supplementary file). A red thrombus was attached to the jailing struts (Fig. 2D-E, red arrows) and prolapsed into the LAD (Fig. $2 \mathrm{~A}-\mathrm{C}$, red arrows).
Aided by strut-automatic-detection software, the longitudinal OCT view (Fig. 3) and the threedimensional (3D)-OCT image fusion (Fig. 4) rendered characteristic stent platform patterns of the previously implanted DES, which could 


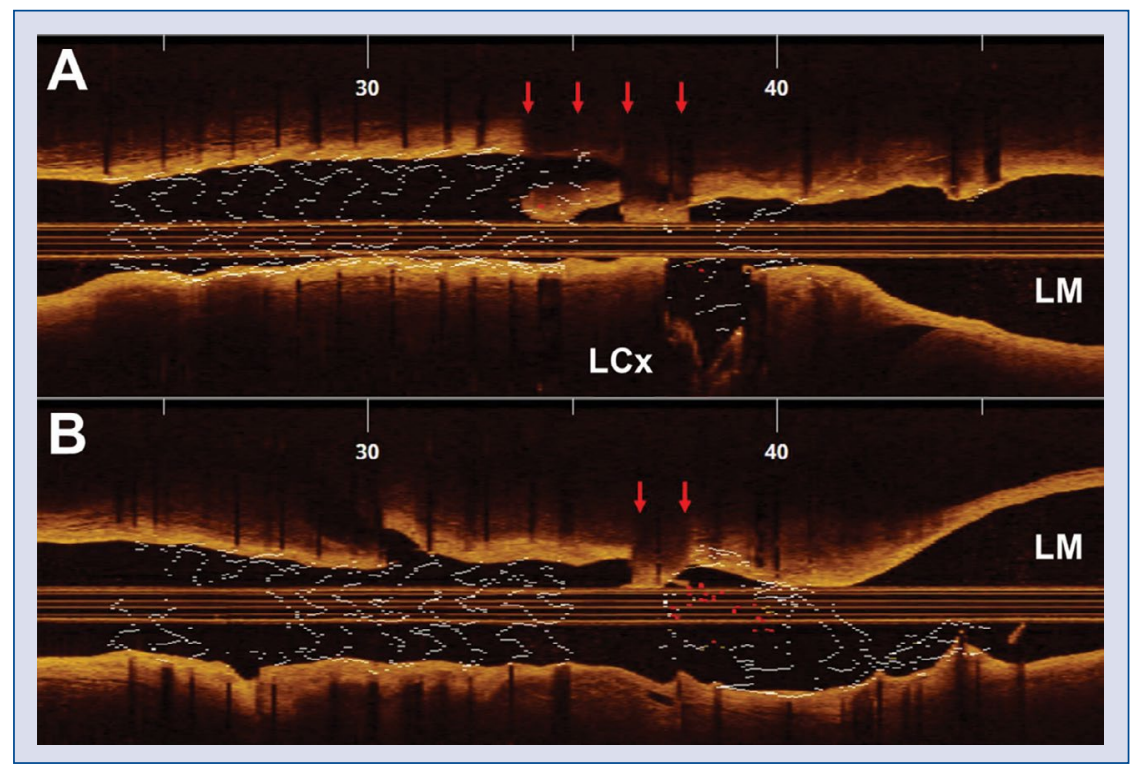

Figure 3. Optical coherence tomography longitudinal views at different rotation positions. The automatic strut detection permits to identify the typical stent platform patterns of a Taxus Liberté (proximal LAD) and a Xience (LCX-LM). A red thrombus (arrows) is attached to the jailing struts of the Xience stent $(\mathbf{A}, \mathbf{B})$, which is crushed against the wall of the left main in its proximal part (B).

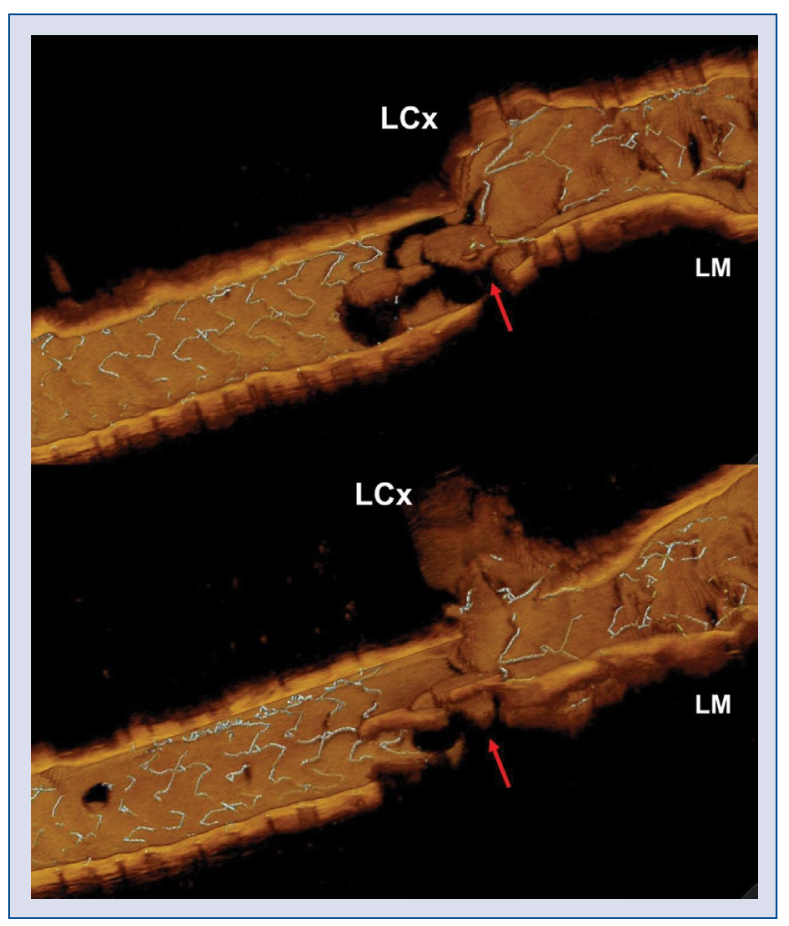

Figure 4. Optical coherence tomography image fusion, rendering the vessel as three-dimensional, at different rotation positions. The automatic strut detection permits to identify the typical stent platform patterns of a Taxus Liberté (proximal LAD) and a Xience (LCX-LM). A red thrombus (arrows) is attached to the jailing struts of the Xience stent, which is crushed against the wall of the left main in its proximal part. be thus identified as a Taxus Liberté (proximal LAD) and a Xience (ostial LCx-LM). The proximal part of the thrombosed Xience was crushed against the wall of the LM, as depicted by the double metallic layer of struts on one sector of the vessel, whilst no layer could be seen on the opposite sector (Fig. 2G-I). The angiographically unapparent stent crushing in the distal left main is most clearly recognisable at first glance in the longitudinal view (Fig. 3), particularly the lower panel (Fig. 3B), and in the 3D-OCT image fusion (Fig. 4). The 3D-OCT image fusion shows in full detail the anchoring of the thrombus to the nonapposed jailing struts of the Xience stent (Fig. 4, red arrows). The thrombus aspirate showed a predominantly red thrombus with very scarce presence of neutrophils (Fig. 5).

A $3 \times 12$ everolimus-eluting stent was implanted in the LAD-LM, with simultaneous kissingballoon inflation and final POT-technique (proximal optimisation technique) in the LM, thus transforming the initially irregular situation of the deformed stent into a mix of reverse-culotte (distally) and crush (proximally), which are biomechanically more predictable scenarios, with an excellent result. No jailing struts can be identified in the final OCT pullback, and malapposition was minimised (Video 3 - see journal website, supplementary file). 


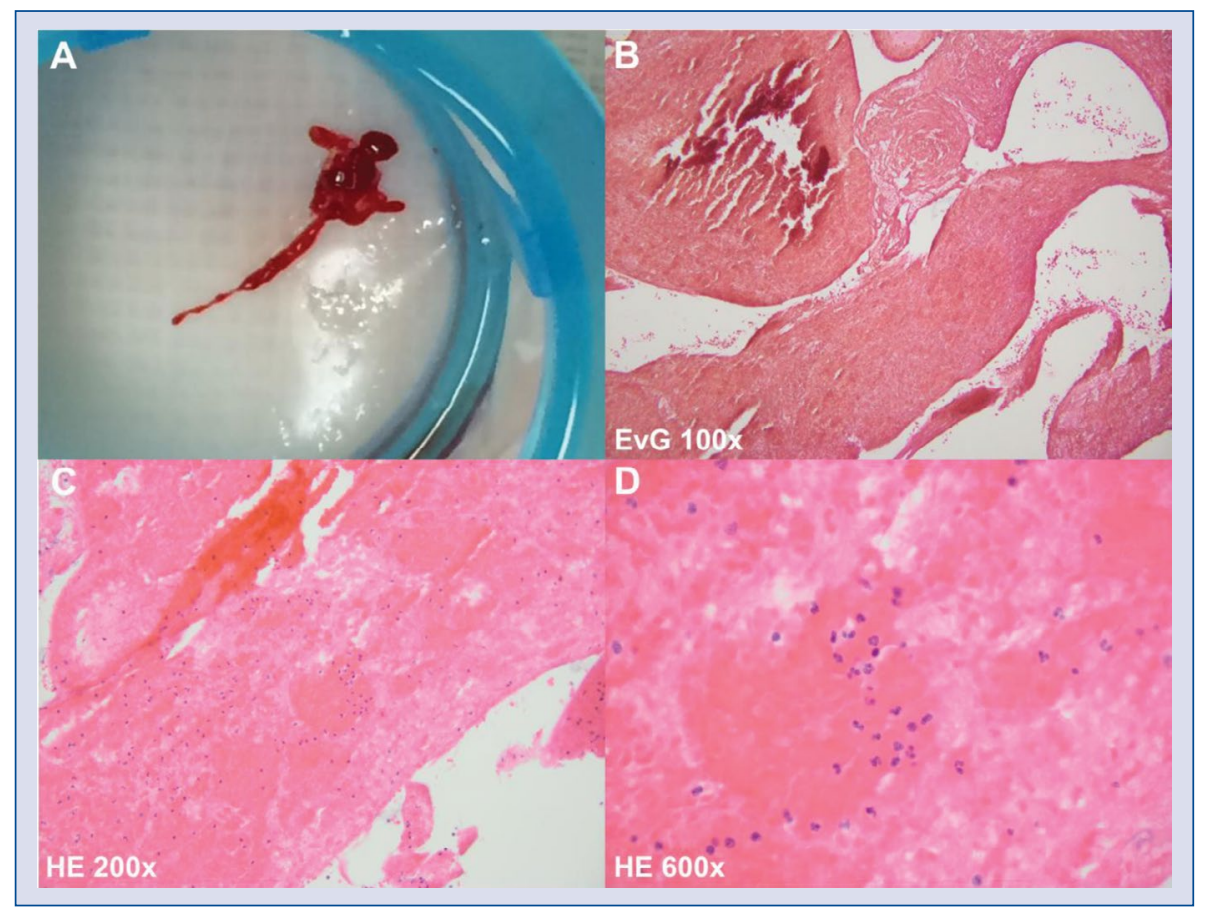

Figure 5. Pathology of the thrombus. The thrombus was harvested by means of aspiration thrombectomy (A). The Elastica-van-Gieson's staining confirms the presence of fibrin and red cells, together with absence of collagen, elastic fibres and elements proper of the vessel wall (B). Haematoxylin-eosin staining shows a red thrombus, as previously depicted in optical coherence tomography, with scarce and predominantly neutrophilic infiltrates (C, D).

\section{Discussion}

Two main pathophysiologic mechanisms of VLST have been described hitherto, namely neoatherosclerosis and vascular toxicity/hypersensitivity vasculitis [7]. Nonetheless both substrates can be reasonably ruled out in the current case after OCT imaging, because no cross-section presents lipid-rich or calcified neointima, thus discarding neoatherosclerosis, or any direct or indirect sign of positive remodeling or late-acquired malapposition, thus discarding vascular toxicity as well. Our case describes however an alternative and plausible mechanism to explain the pathogenesis of the thrombus. Albeit inadvertent in the coronary angiography, the OCT imaging unravels a poorly implanted stent in the LCx, extending up to the LM and crushed against the LM wall, probably due to accidental abluminal wiring during the implantation. The stent structure is severely distorted, leaving a double metallic layer that jails the ostium of the LAD and poses a substrate of gross malapposition in the LM. These scenario is a known predisposing factor for thrombotic complications, at least theoretically, because it entails higher risk of delayed healing $[1,2]$, alters severely the coronary flow and induces high shear stress rates at the metallic surface of some struts, that might activate the platelets and the coagulation cascade [3]. Therefore, having reasonably ruled out other causes of VLST, we can carefully claim that the most likely and most plausible mechanism for the thrombosis in this case are the flow disturbances induced by the gross malapposition and the jailing struts of the suboptimally implanted stent in the LCx. As additional evidence supporting this mechanism, the OCT has clearly identified the anchoring point of the thrombus in the centre of the metallic mesh jailing the ostium of the LAD, which is the region where the neointimal coverage should be poorest [2] and the shear stress rate highest [3] according to previous studies, therefore where the thrombotic diathesis should be highest according to the predictions of the shear stress model of flow disturbances induced by detached struts. To the best of our knowledge this is the first report linking so clearly a case of VLST with predisposing mechanical factors in a poorly implanted stent. Our paradigmatic case complements previous case series of VLST, studied in full detail with 
invasive imaging and histopathologic analysis of the thrombus, in which this association could not be demonstrated [7].

The histopathology of the thrombus suggests also a differentiated pathogenesis from that described in cases of vascular toxicity or vasculitis. Delayed hypersensitivity reactions recruiting predominantly eosinophils, lymphocytes, plasma cells, palisading macrophages and giant cells have been described in cases of VLST of both first-generation $[4,5]$ and second-generation DES [6]. These inflammatory infiltrates could be observed in the stented vessel wall $[4,6]$, but also in the harvested thrombus [5]. A granulocytic infiltrate rich in eosinophils is more often observed in sirolimus-eluting stents than in paclitaxel-eluting stents undergoing VLST [5]. Nonetheless, instead of this profuse eosinophilic infiltrate, the current case shows scarce neutrophilic presence in the aspirated thrombus, that cannot be properly considered an inflammatory infiltrate. It might quantitatively correspond to the circulating granulocytes that get trapped in the thrombus mesh during its formation, thus suggesting a different pathogenesis, with negligible role of inflammation.

Furthermore, the stent thrombosis in the current case can hardly be explained by the properties of the DES, because the thrombus originates from the second-generation DES (Xience, Abbott vascular, Santa Clara, CA), which has a more biocompatible and hemocompatible profile than the first-generation paclitaxel-eluting stent (Taxus, Boston Scientific, Natick, MAD, USA). The paradoxal occurrence of VLST against aprioristic probability in the most hemocompatible DES highlights the importance of the biomechanical factors, since the Taxus stent was optimally deployed and covered, whilst the Xience stent was severely distorted, as previously described.

The case offers a novel insight into the pathogenesis of very late stent thrombosis, describing an additional mechanism insufficiently addressed in previous publications and confirming the thrombotic propensity of mechanical factors, like malapposition and jailing. Furthermore, the case illustrates also the instrumental role of OCT to tailor the therapy in cases of device failure, an indication currently accepted as IIa in current European Society of Cardiology guidelines. Finally, this case is the first scientific report pointing the ability of $3 \mathrm{D}-\mathrm{OCT}$ to identify the stent type whenever this information is missing.

\section{Conflict of interest: None declared}

\section{References}

1. Gutiérrez-Chico JL, Regar E, Nüesch E, et al. Delayed coverage in malapposed and side-branch struts with respect to well-apposed struts in drug-eluting stents: in vivo assessment with optical coherence tomography. Circulation. 2011; 124(5): 612-623, doi:10.1161/CIRCULATIONAHA.110.014514, indexed in Pubmed: 21768536.

2. Gutiérrez-Chico JL, Wykrzykowska J, Nüesch E, et al. Vascular tissue reaction to acute malapposition in human coronary arteries: sequential assessment with optical coherence tomography. Circ Cardiovasc Interv. 2012; 5(1): 20-29, doi: 10.1161/CIRCINTERVENTIONS.111.965301, indexed in Pubmed: 22319063.

3. Foin N, Gutiérrez-Chico JL, Nakatani S, et al. Incomplete stent apposition causes high shear flow disturbances and delay in neointimal coverage as a function of strut to wall detachment distance: implications for the management of incomplete stent apposition. Circ Cardiovasc Interv. 2014; 7(2): 180-189, doi: 10.1161/CIRCINTERVENTIONS.113.000931, indexed in Pubmed: 24642998.

4. Virmani R, Guagliumi G, Farb A, et al. Localized hypersensitivity and late coronary thrombosis secondary to a sirolimuseluting stent: should we be cautious? Circulation. 2004; 109(6): 701-705, doi: 10.1161/01.CIR.0000116202.41966.D4, indexed in Pubmed: 14744976 .

5. Cook S, Ladich E, Nakazawa G, et al. Correlation of intravascular ultrasound findings with histopathological analysis of thrombus aspirates in patients with very late drug-eluting stent thrombosis. Circulation. 2009; 120(5): 391-399, doi: 10.1161/CIRCULATIONAHA.109.854398, indexed in Pubmed:19620501.

6. Otsuka F, Yahagi K, Ladich E, et al. Hypersensitivity reaction in the US Food and Drug Administration-approved second-generation drug-eluting stents: histopathological assessment with ex vivo optical coherence tomography. Circulation. 2015; 131(3): 322-324, doi:10.1161/CIRCULATIONAHA.114.012658, indexed in Pubmed: 25601953.

7. Guagliumi G, Sirbu V, Musumeci G, et al. Examination of the in vivo mechanisms of late drug-eluting stent thrombosis: findings from optical coherence tomography and intravascular ultrasound imaging. JACC Cardiovasc Interv. 2012; 5(1): 12-20, doi: 10.1016/j.jcin.2011.09.018, indexed in Pubmed: 22230145. 